\title{
Bioinformatics Analysis of Key Genes and Pathways of Cervical Cancer
}

This article was published in the following Dove Press journal:

OncoTargets and Therapy

\author{
Huan Chen (1) \\ Xi Wang' \\ Huanhuan Jia ${ }^{2}$ \\ Yin $\mathrm{TaO}^{3}$ \\ Hong Zhou ${ }^{3}$ \\ Mingyuan Wang ${ }^{4}$ \\ Xin Wang' \\ Xiaoling Fang $(\mathbb{D})^{\prime}$ \\ 'Department of Obstetrics and \\ Gynecology, The Second Xiangya \\ Hospital of Central South University, \\ Changsha, Hunan 4100II, People's \\ Republic of China; ${ }^{2}$ Guangdong \\ Laboratory Animals Monitoring Institute, \\ Guangdong Key Laboratory of \\ Laboratory Animals, Guangzhou, \\ Guangdong 510663, People's Republic of \\ China; ${ }^{3}$ Department of Obstetrics and \\ Gynecology, Zhu Zhou Hospital Affiliated \\ to Xiangya School of Medicine, CSU, \\ Zhuzhou, Hunan 4I2007, People's \\ Republic of China; ${ }^{4}$ Department of \\ Pathophysiology, School of Basic Medical \\ Science, Central South University, \\ Changsha, Hunan, 410013, People's \\ Republic of China
}

Background and Objective: Globally, cervical cancer (CC) is the fourth most common cancer affecting women. Although effective screening reduces its incidence, it remains one of the most serious cancers threatening the health of women. Therefore, the purpose of this study is to find new genes that can be used as potential biomarkers for the prognosis of CC. Methods and Results: After downloading three datasets such as GSE6791, GSE63678, and GSE63514 from the Gene Expression Omnibus (GEO), we combined the expression matrixes and analyzed them to obtain the differential expressed genes (DEGs). Next, using the STRING website, we performed the protein interaction network analysis. Subsequently, hub genes were screened using the $\mathrm{R}$ and Cytoscape software. Then, the expression difference and survival analyses of the hub genes were confirmed using GIPIA. Here, we established that the KNTC1 gene was correlated to the overall survival prognosis of CC. Besides, the expression of the KNTC1 gene in the GSE63514 dataset was significantly different from that of the normal cervix, cervical pre-cancerous lesions, and $\mathrm{CC}$. Consequently, immunohistochemistry analysis showed that the results have a definite diagnostic value.

Conclusion: The KNTC1 gene could be linked with the pathophysiology of CC and maybe one of the early diagnostic markers for the diagnosis of cervical pre-cancerous lesions.

Keywords: cervical cancer, bioinformatics analysis, differentially expressed gene, pathways, KNTC1

\section{Introduction}

Worldwide, ${ }^{1}$ cervical cancer (CC) is the fourth most common cancer affecting women. It has a morbidity of 371,000 cases and a mortality rate of 190,000 cases annually. ${ }^{2}$ Though its incidence has dropped year after year in developed countries, it is escalating in developing countries. Studies have shown that approximately $90 \%$ of CC-related deaths have occurred in developing countries. On the other hand, ${ }^{3}$ cervical intraepithelial neoplasia (CIN) is a pre-cancerous potential transformation of squamous cells on the surface of the cervix. Following an abnormal hyperplasia degree, it is categorized into three different types such as CIN I, II, and. The CIN I type is also called a low-grade squamous intraepithelial lesion (LSIL), and CIN II and CIN III are the high-grade squamous intraepithelial lesion (HISL). ${ }^{4}$ Typically, it takes 8 years on average for CIN to develop into CC. According to literature, 5,6 squamous cell carcinoma accounts for about 80 to $90 \%$ of the total CC cases. First, pre-cancerous lesions develop from LSIL to HISL, and subsequently to squamous cell carcinoma. However, ${ }^{7,8}$ the CC pathogenesis is unclear and could be caused by recurrent genetic alterations, microenvironment, lifestyle, smoking, etc. ${ }^{9-12}$
Correspondence: Xiaoling Fang

Tel +86 I3973 I28345

Email fxlfx10510@csu.edu.cn
OncoTargets and Therapy 2020:13 13275-13283

13275 
Different studies have revealed that persistent HPV infection is the main trigger of CC. However, only a small proportion of HPV infection patients will eventually develop CC. ${ }^{13}$ Etiological studies have shown that the $\mathrm{CC}$ occurrence is a complicated process that involves multiple factors, and its molecular mechanism has not yet been fully elucidated. Therefore, there is a need to find an indicator that predicts the pre-cancerous lesion which leads to cancer.

Currently, ${ }^{14}$ the profile data of gene expression is available, and its data analysis using bioinformatics has become a promising area of new research. Studies have shown that ${ }^{15}$ bioscience research increasingly depends on bioinformatics to efficiently organize and analyze biological data and experimental results, which ${ }^{16}$ provides a theoretical basis for a profound understanding of molecular events occurring in human biology and disease. Besides, ${ }^{17,18}$ it has been extensively used to study molecular changes in tumor progression to better comprehend the development of human carcinogenesis development and to discover novel predictive cancer markers.

GEO (https://www.ncbi.nlm.nih.gov/geo) is a publicly available database that supports a large number of microarray expression data sets. Here, we downloaded and analyzed three already established microarray datasets such as $^{19}$ GSE6791, ${ }^{20}$ GSE63678, ${ }^{21}$ GSE63514 from the GEO database. Subsequently, we performed an analysis of DEGs that are present in normal cervical and CC tissues using the R statistical software. Concurrently, we verified differential gene expression using different databases like ${ }^{22}$ the online analysis tool GIPIA, based on GTEx, and TCGA databases, which contained 306 CC samples and 13 normal cervical tissues. Next, the DEGs protein interactions were analyzed on the STRING online website. Consequently, our study established that the KNTC1 expression levels were substantially related to the OS of $\mathrm{CC}$ patients and it was quite different in normal cervix, cervical pre-cancerous lesions, and CC. Lastly, we used immunohistochemistry to verify this finding and we concluded that the KNTC1 gene is a prospective key gene for $\mathrm{CC}$ pre-cancerous lesions and prognosis.

\section{Materials and Methods}

\section{Microarray Data}

We downloaded ${ }^{19-21}$ three CC GEO datasets, including GSE6791, GSE63678, and GSE63514, from the Gene Expression Omnibus (http://www.ncbi.nlm.nih.gov/gds).
Table I Dataset Information

\begin{tabular}{|l|l|l|l|l|}
\hline GEO & Platform & Sample & Normal & Tumor \\
\hline GSE63678 & GPL57I & Cervix & 5 & 5 \\
GSE679I & GPL570 & Cervix & 8 & 20 \\
GSE63514* & GPL570 & Cervix & 24 & 28 \\
\hline
\end{tabular}

Notes: *The datasets also contain I4 CIN I, 22 CIN II, 40 CIN III samples.

Table 1 illustrates the details of GEO cervical cancer data.

\section{Data Normalization and Exploration}

We first downloaded a series of matrix files, and the platform was converted using the Perl programming and scripting language software. Next, the ID corresponding to the probe name was converted into an international standard name for genes (symbols) and then merged into a CSV file. ${ }^{23}$ Subsequently, we eliminated the batch effect using the Surrogate Variable Analysis (SVA) package of Bioconductor (http://www.biocon ductor.org/). ${ }^{24}$ Consequently, we selected the DEGs using the limma package. Lastly, we selected DEGs, using a cutoff criteria of adj.P.Val $<0.05$ and $\mid \log 2$ (fold change) $\mid>2$.

\section{Protein-Protein Interaction (PPI) Network}

We analyzed the PPI pairs of the screened DEGs using ${ }^{25}$ the online database STRING version 11 (https://string-db. org/). Next, the pairs with a combining score $>0.4$ were used for the PPI network construction. Then, we removed the partial and isolated connected nodes, constructed a complex network of DEGs, downloaded the excel of string-interactions, and saved them in a CSV file format. Lastly, the DEGs expression and String-interactions files were imported into Cytoscape software for further processing, whereby we constructed a network and analyzed the interactive relationship of the candidate DEGs encoding proteins in $\mathrm{CC}$.

\section{DEGs Basic Expression in Normal and Cancer Tissues}

Here, $^{22}$ the online website GEPIA was selected as a validation set for Screening the DEGs. GEPIA is a newly developed interactive web server that has a standard processing pipeline and was used to analyze the sequenced RNA expression data of 9736 tumors and 8587 normal samples from the TCGA and GTEx projects, that were initially based on the UCSC Xena Project (http://xena.ucsc.edu). 


\section{Biomarker Discovery for Early Diagnosis}

The potential application for early diagnosis was evaluated using GSE63514 datasets to perform microarrays prediction analysis by comparing normal to LISL, LISL to HISL, and HISL to CC samples.

\section{Tissue Collection}

From August 2020 to September 2020, normal cervical, CIN I-III, and cervical squamous cell carcinoma tissue samples were acquired from Zhuzhou Hospital which is allied to Xiangya Medical College. All specimens were subjected to immunohistochemical evaluation and confirmed by two independent pathologists. This study was approved by the research and clinical trial ethics committee of Zhuzhou Hospital, and all eligible participants provided written informed consent. All clinical procedures were performed per the ethical standards of the Declaration of Helsinki guidelines and relevant policies in China.

\section{Immunohistochemistry}

To validate Kinetochore Associated protein 1 (KTNC1), immunohistochemical staining was performed on the specimen sections of normal, LSIL, HSIL, and CC samples. The following steps were followed: section dewaxing, antigen repair, KTNC1 antibody (Abcam, Cambridge, UK) incubation, and secondary antibody incubation. ${ }^{26}$

\section{Results}

\section{Identification of DEGs in CC}

Our study involved 39 healthy individuals and 51 patients with $\mathrm{CC}$ and their three mRNA expression profiles were merged into one dataset. Using a cutoff criteria of adj.P.Val $<0.05$ and $|\log \mathrm{FC}|>2$, we extracted a total of 161 differentially expressed probesets (63 downregulated and 98 upregulated) from the expression profile datasets. The volcano map illustrates the differential genes (Figure 1A) whereas the heatmap shows differentially expressed probe sets between normal versus cancer tissues (Figure 1B).

\section{Pathway Analysis}

Here, enrichment analyses were performed for Gene Ontology gene sets (GO) using the ClusterProfiler ${ }^{27}$ and the org.Hs.eg.db packages ${ }^{28}$ As illustrated in Figure 1C, it was observed that the $161 \mathrm{CC}$ intrinsic genes were mostly involved in the cell cycle through processes such as DNA replication and recombination, RNA metabolism, purine, and pyrimidine metabolism. Besides, by utilizing the
ClusterProfiler package, ${ }^{27}$ a similar analysis was performed in the Kyoto Encyclopedia of Genes and Genomes (KEGG) database. However, only one pathway was found to be involved in DNA replication.

\section{PPI Network Construction}

At a combined score $>0.4$, Figure $2 \mathrm{~A}$ shows a total of 136 DEGs (92 upregulated and 44 downregulated genes) from the 161 commonly altered DEGs that were filtered into the DEGs PPI network and they contained 136 nodes and 1363 edges. Subsequently, the node degree ${ }^{29}$ was estimated using the cytohubba app in Cytoscape software. Next, as illustrated in Figure 2B, the most significant 20 node degree genes were selected as the hub nodes since they could play a vital role in the progression of $\mathrm{CC}$.

\section{Key Gene Signatures Identification in CC}

To calculate the counts between DEGs, we used the Stringinteractions file, and as illustrated in Figure 2C, we selected the top 20 genes. Subsequently, we overlapped the 20 genes with hub nodes, and as demonstrated in Figure 2D, we selected the following key genes; MELK, CDK1, BUB1B, NCAPG, KIF11, PBK, TOP2A, TTK, DLGAP5, ASPM, DTL, CCNB1, CDC6, RAD51AP1, KNTC1, KIF15, and $N D C 80$. Also, as shown in Figure $3 \mathrm{~A}$ and $\mathrm{B}$, univariate analysis $(\mathrm{P}<0.05)$ showed that the expression levels of $K N T C 1$ and $T O P 2 A$ were significantly related to the OS of the CC patients. The high expression of $K N T C 1$ and TOP $2 A$ could lead to a high rate of survival. Notably, in CC tissues, the expression of these two genes was significantly higher than that of normal tissues $(\mathrm{P}<0.01$; Figure $3 \mathrm{C}$ and $\mathrm{D})$. However, its expression was not correlated to the $\mathrm{CC}$ grade. Taken together, the high level of these two genes could represent the significant prognostic factor that predicts the survival of CC patients.

\section{Validation of Hub Genes}

To identify biomarkers for early discovery, we adopted a four-stage approach. Here, we first extracted the KNTC1 expression profiles from GSE6791 datasets. These datasets contained 24 normal cervix,14 CIN I, 22 CIN II, 40 CIN III, and 28 CC tissues. Next, we compared the expression profiles of normal, LSIL (CIN I), HSIL (CIN II, III), and CC samples using the Wilcox test. As illustrated in Figure 4A, we established that the KNTC1 expression is significantly different in normal cervix, cervical pre-cancerous lesions, and in $\mathrm{CC}$ tissues. 


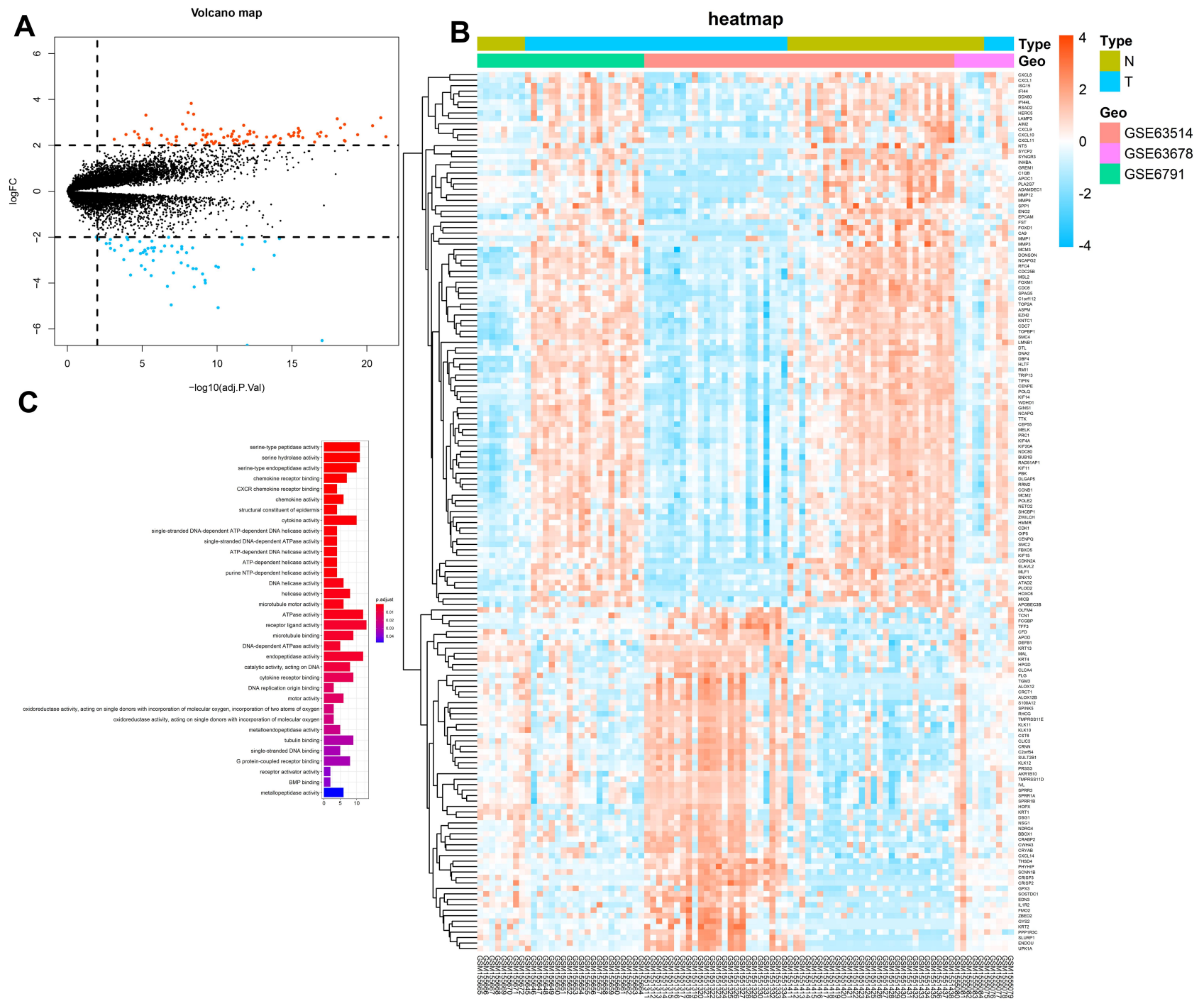

Figure I (A) Volcano plot of three datasets (GSE639I4, GSE63678, GSE679I), orange nodes represent DEGs with logFC >2 and p-value of <0.05. Blue nodes represent DEGs with logFC $<-2$ and p-value of $<0.05$. (B) A heat map of all DEGs of three datasets, Each column represents a sample and each row represents one gene. The gradual color ranging from blue to orange represents the gene expression changing from downregulation to upregulation. The letter $\mathrm{N}$ represents normal tissue, and the letter T represents cancer tissue. (C) GO enrichment analysis of DEGs.

Besides, we performed immunochemical staining for KNTC1 in the normal cervix, LSIL, HSIL, and CC tissue, which could show the following; weak staining of the normal cervical tissue, HISL was significantly enhanced than LSIL, and the cervical squamous cell carcinoma was also significantly enhanced than normal cervical tissues (Figure 4B-F).

\section{Discussion}

Studies have shown that hub genes play critical roles during the different cancer progression stages. Although several studies on the development of $\mathrm{CC}$ are available, there is a need to establish more research to identify driver genes and candidate drugs for the treatment of
CC. In a nutshell, our study integrated three different gene profile datasets, utilized bioinformatics techniques to analyze these datasets, and identified a total of 161 frequently changed DEGs (98 upregulated and 63 downregulated). The pathway enrichment analysis showed that cell cycle and DNA replication pathways were over-represented among the upregulated genes. Besides, the PPI network was constructed and it included 136 nodes and 1363 edges. Consequently, 16 key genes were determined and chosen for subsequent survival analysis. Here, we observed that the KNTC1 gene was related to the prognosis of $\mathrm{CC}$ patients. Also, small molecules that could provide new insights into the therapeutic studies of $\mathrm{CC}$ were identified. 

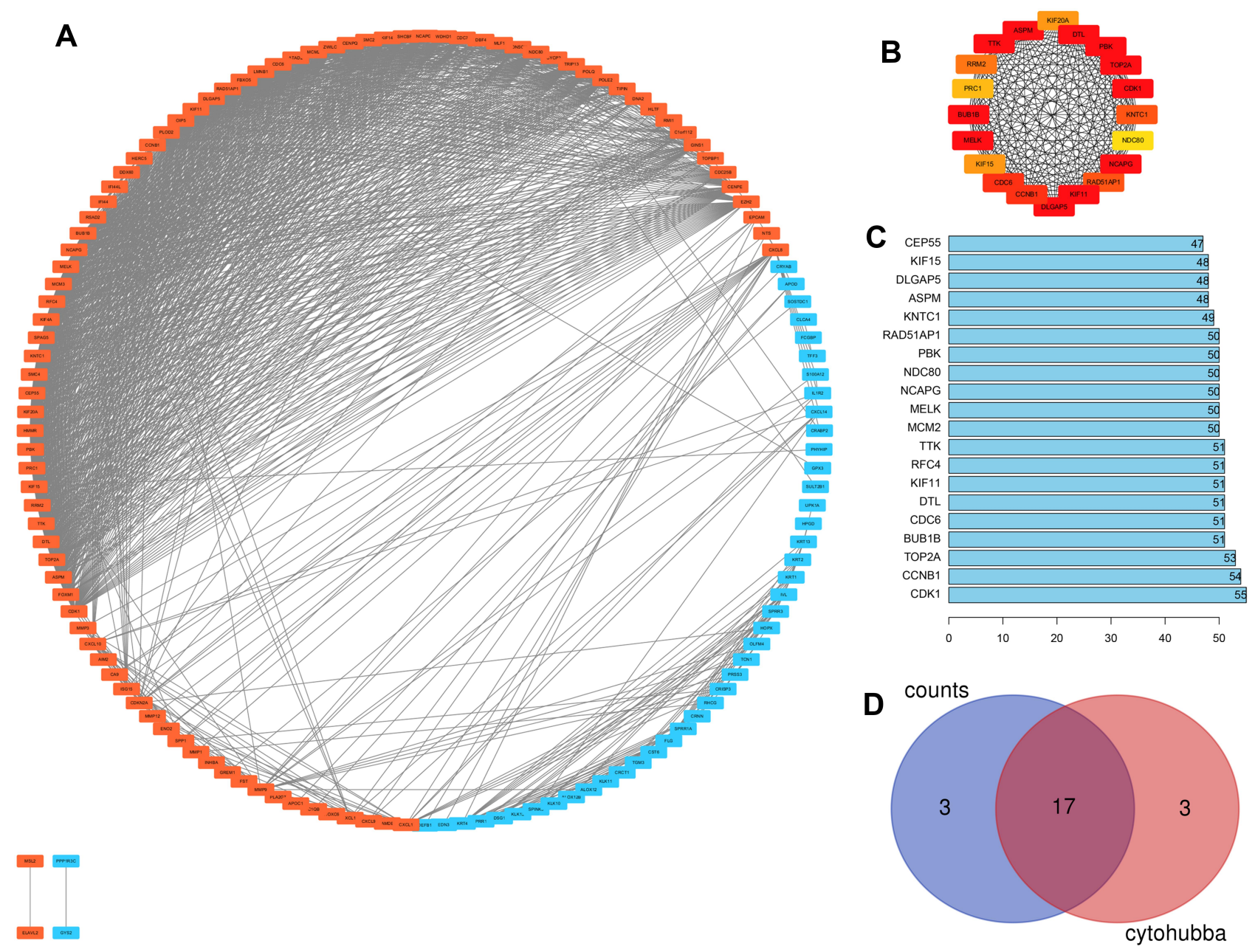

Figure 2 (A) PPI of DEGs, orange nodes for upregulation genes, blue nodes for downregulation genes. (B) The most significant 20 node degree genes calculated by cytohubba APP of cytoscape. (C) The top 20 genes selected by using the String-interactions file to calculated the counts between DEGs. (D) The Venn diagram of the top 20 degree genes and the top 20 counts genes.

Studies have shown that the Kinetochore-related protein 1 (KNTCl) gene is disseminated in the cytoplasm, nucleus, chromosomal centromere, centromere, cytoskeleton, and in the spindle fiber. Its distribution is different with the cell cycle, and it is evenly distributed throughout the cytoplasm and nucleus during its interphase stage. Besides, a small part of the entire cell is concentrated on the centromere from the early stage of cell division to the late stage of the middle and early stages. ${ }^{30}$ Previous studies have also shown that by the middle of cell division, it was identified at the centromere, along the spindle fiber, majorly at the pole. However, from the late stage of splitting to the final stage, the centromere or spindle fiber could no longer be detected, but it still existed at the pole of the spindle. ${ }^{31}$ The KNTC1 gene acts on the G2-M phase of the cell cycle and several studies have established that it is involved in the cell cycle process, hence participating in the occurrence of disease. Moreover, it has a significant association with the progression of liver fibrosis. ${ }^{32}$ Notably, the level of this gene was lower in mycoplasma pneumonia, than in the healthy control group.

Also, KNTC1 has been investigated in various human malignancies and has been associated with tumor histopathological grade. ${ }^{33}$ A study explored the expression levels of KNTC1 mRNA in three esophageal squamous cell carcinoma (ESCC) cell lines and established that all the tested cell lines showed that it is positively expressed. However, knocking out this gene can effectively inhibit cell viability and increase apoptosis. From the online ESCC dataset, a gene set enrichment analysis showed that overexpression of the KNTC1 gene is related to increased mitotic spindles and hypoxic pathways, and 

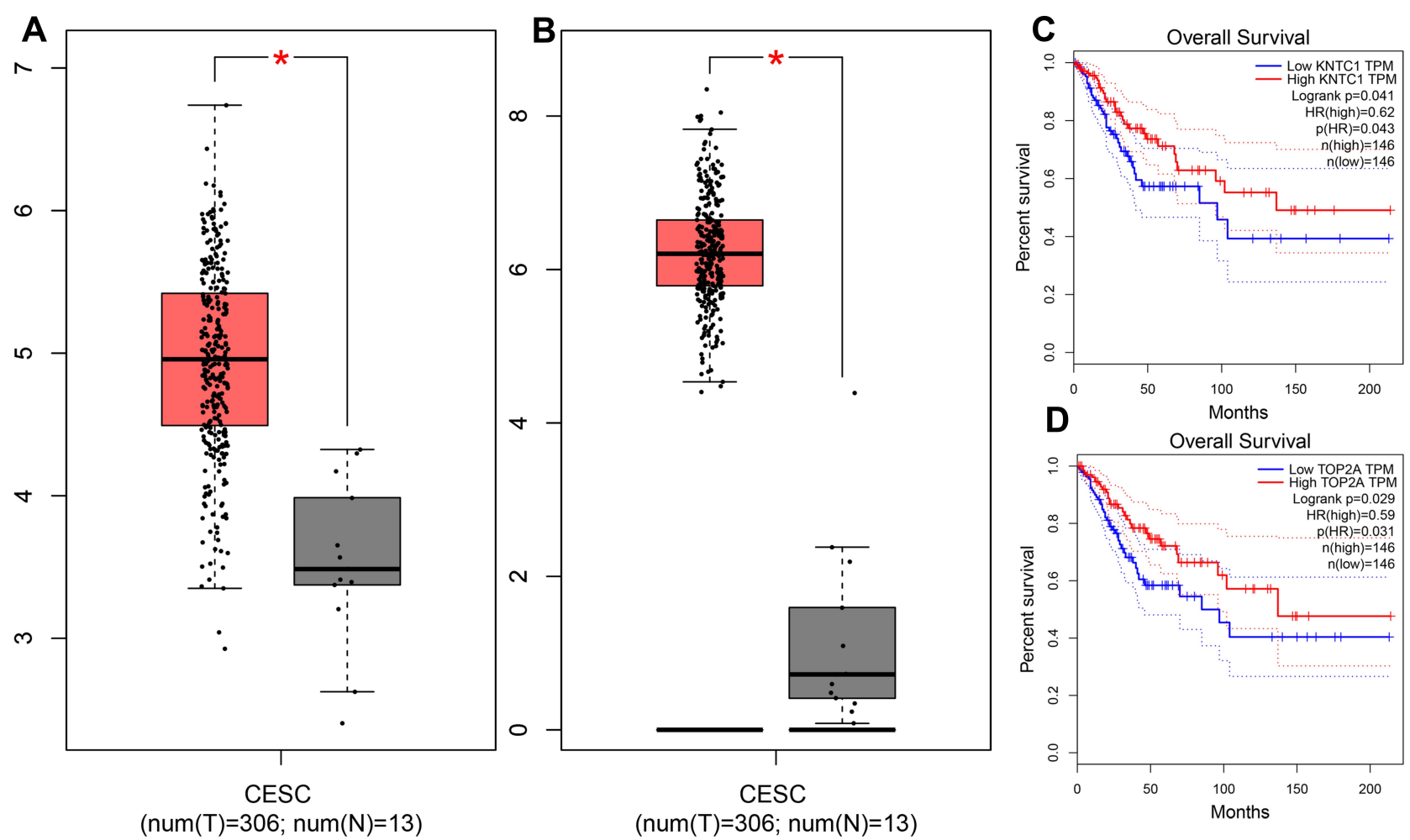

Figure 3 (A and B) Expression boxplots of KNTCI/TOP2A genes using GEPIA website $(\mathbf{P}<0.05)$. (C and D) Survival analysis. Expression level of KNTCI/TOP2A was significantly related to the overall survival of patients with cervical squamous cancer $(P<0.05)$.

with decreased DNA repair and mismatch repair pathways. KNTC1 could play an important role in regulating the viability and apoptosis of ESCC cells and may perhaps become a novel therapeutic strategy for ESCC. ${ }^{34}$ Previous studies have shown that in patients with oral squamous cell carcinoma, the transcriptional activity of exact cell cycle genes, like the KNTC1, changes with the size of the tumor, which could reflect the evolution and adaptation of tumor molecules to the microenvironment. Also, compared with the healthy control group, the KNTCl expression in neuroblastoma samples increased statistically. ${ }^{35}$ Although the increase in gene expression level is minimal, it can also play a significant role in promoting neuroblastoma. ${ }^{36}$ Furthermore, the KNTCl frameshift mutation could cause signal damage/repair regulation and promote gastric cancer (GC) and colorectal cancer (CRC) with microsatellite instability (MSI) development. ${ }^{37-39}$ Several bioinformatics studies have also established that the KNTC1 gene could be one of the vital genes associated with cancer development, including Hepatocellular carcinoma (HCC), Pancreatic cancer (PC), and nasopharyngeal carcinoma (NPC). ${ }^{40}$ In mitotic checkpoints, the KNTC1 gene is a key component that ensures proper chromosome separation during cell division. These processes are both critical, highly ordered biological processes that depend on numerous evolutionarily conserved protein complexes. ${ }^{41}$ A study showed that many proteins that regulate the mitotic process are overexpressed in human malignancies than in their normal counterparts, and some are conveyed to be oncogenes. ${ }^{42}$ To date, the different KNTC1 gene functions include: recruiting cytoplasmic dynein to centromeres; participating in the chromosomes mitochondrial movement during mitosis; maintaining functional metaphase checkpoints; ${ }^{40}$ regulating exit from mitosis; ${ }^{43}$ proteincontaining complex assembly; involved in cell division; separation of sister chromatids; resolution of sister chromatid cohesion; EML4 and NUDC in mitotic spindle formation; ${ }^{44}$ Amplification of signal from unattached kinetochores through a MAD2inhibitory signal; and mitotic Prometaphase.

Clinically, this study has great significance for a better understanding of the development and prognosis of CC. However, it is imperative to perform further clinical research to ascertain whether these genes can be used as biomarkers for the diagnosis and prognosis of CC. 


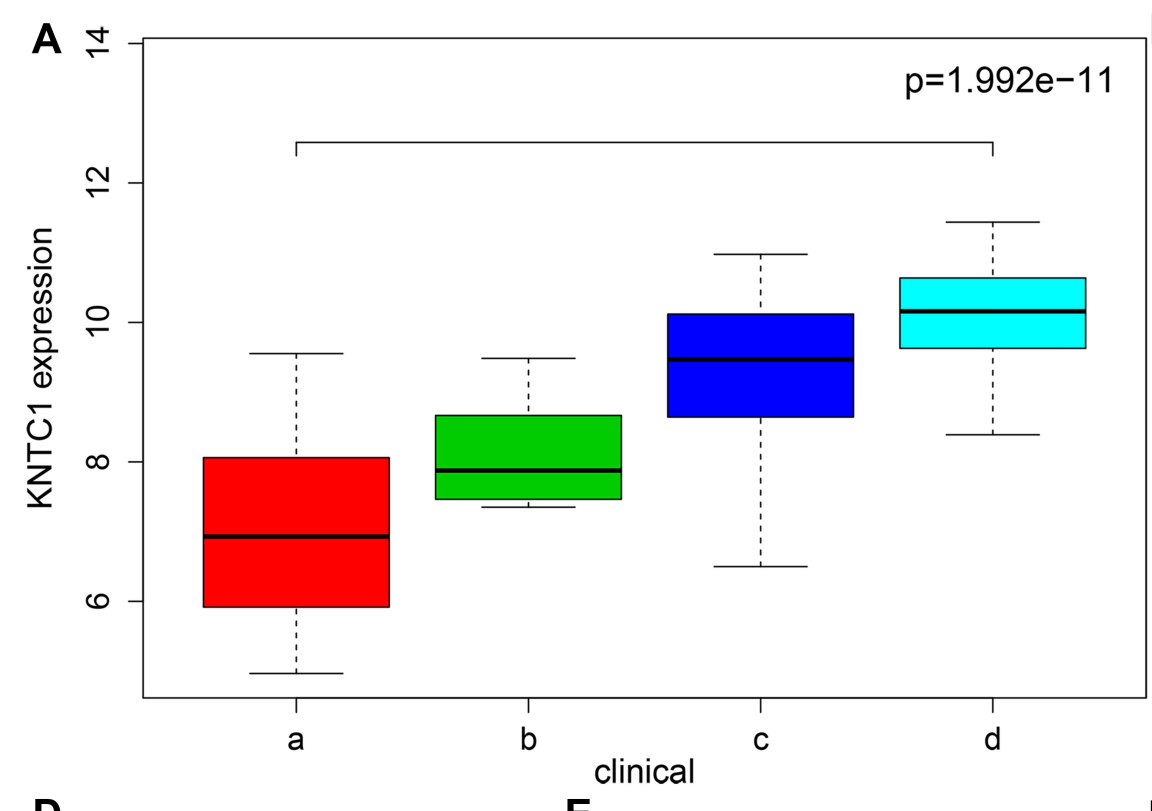

D

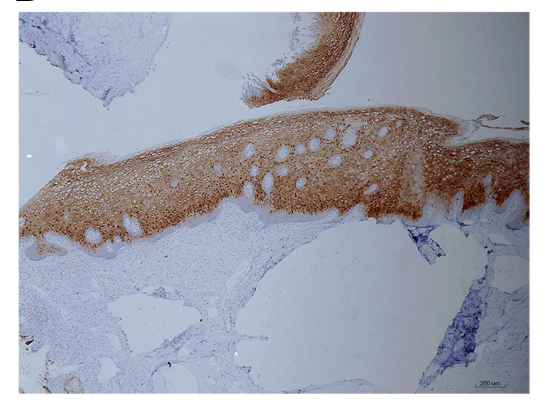

E

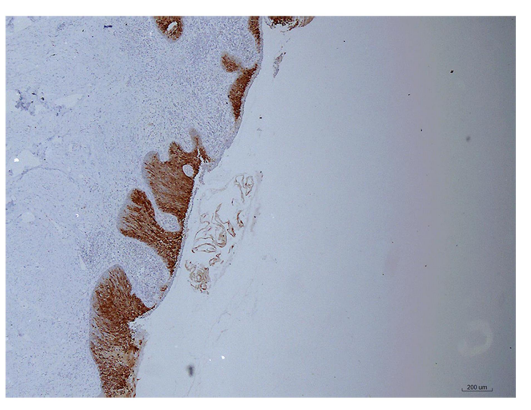

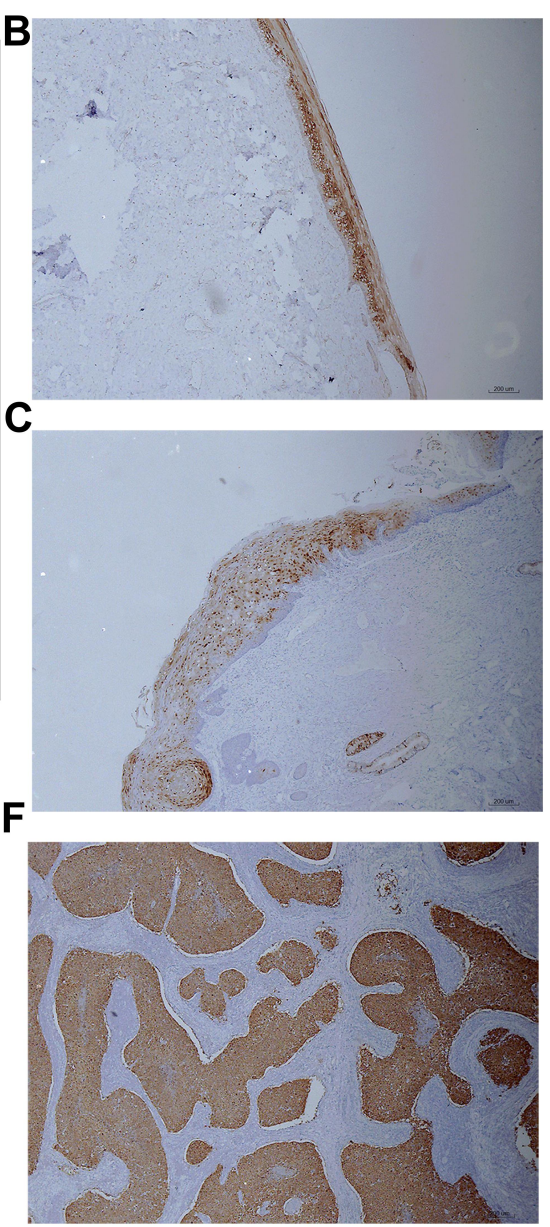

Figure 4 (A) The expression of KNTCI of normal cervix, cervical precancerous lesions and CC in Dataset GSE679I.a represents normal cervix, b represents LSIL (CIN I), c represents HSIL (CIN II), d represents cervical squamous cell carcinoma. (B-F) Immunohistochemical staining of KNTCI gene in normal cervical, LSIL (CIN I), HSIL (CIN II, III) and cervical squamous cell carcinoma specimens.

\section{Conclusion}

Taken together, our study has established that the KNTCl gene is closely related to the poor prognosis of $\mathrm{CC}$. Its expression is also different in normal cervical, precancerous lesions, and cancer tissues. Therefore, it could be an important biomarker for early diagnosis and prediction of cervical pre-cancerous lesions.

\section{Disclosure}

The authors report no conflicts of interest for this work.

\section{References}

1. Kassa U, Dile LS, Zenebe WM. Precancerous lesions of cervix among women infected with HIV in referral hospitals of Amhara region, northwest Ethiopia: a cross sectional study. Afr Health Sci. 2019;19 (1):1695-1704. doi:10.4314/ahs.v19i1.46

2. Banno K, Iida M, Yanokura M, et al. MicroRNA in cervical cancer: oncomiRs and tumor suppressor miRs in diagnosis and treatment. TheScientificWorldJournal. 2014;2014:178075. doi:10.1155/2014/ 178075
3. Cheng X, Feng Y, Wang X, Wan X, Xie X, Lu W. The effectiveness of conization treatment for post-menopausal women with high-grade cervical intraepithelial neoplasia. Exp Ther Med. 2013;5(1):185-188. doi:10.3892/etm.2012.748

4. Li L, Ding L, Gao $\mathrm{T}$, et al. Association between vaginal micro-environment disorder and cervical intraepithelial neoplasia in a community based population in China. J Cancer. 2020;11 (2):284-291. doi:10.7150/jca.35022

5. Ramírez A, Vera E, Gamboa-Domínguez A, Lambert P, Gariglio P, Camacho J. Calcium-activated potassium channels as potential early markers of human cervical cancer. Oncol Lett. 2018;15(5):72497254. doi:10.3892/ol.2018.8187

6. Zur Hausen H. Papillomaviruses and cancer: from basic studies to clinical application. Nat Rev Cancer. 2002;2(5):342-350. doi:10.1038/nrc798

7. de Freitas AC, Gurgel AP, Chagas BS, Coimbra EC, Do Amaral CM. Susceptibility to cervical cancer: an overview. Gynecol Oncol. 2012;126(2):304-311. doi:10.1016/j.ygyno.2012.03.047

8. Wani K, Nair CK. Genetic alterations in cervical cancer. Indian J Exp Biol. 2003;41(8):789-796.

9. Walboomers JM, Jacobs MV, Manos MM, et al. Human papillomavirus is a necessary cause of invasive cervical cancer worldwide. J Pathol. 1999;189(1):12-19. doi:10.1002/(SICI)1096-9896(199909) 189:1<12::AID-PATH431>3.0.CO;2-F

10. Drain PK, Holmes KK, Hughes JP, Koutsky LA. Determinants of cervical cancer rates in developing countries. Int J Cancer. 2002;100 (2):199-205. doi:10.1002/ijc.10453 
11. McMurray HR, Nguyen D, Westbrook TF, McAnce DJ. Biology of human papillomaviruses. Int $J$ Exp Pathol. 2001;82(1):15-33. doi:10.1046/j.1365-2613.2001.00177.x

12. Scheurer ME, Danysh HE, Follen M, Lupo PJ. Association of traffic-related hazardous air pollutants and cervical dysplasia in an urban multiethnic population: a cross-sectional study. Environ Health. 2014;13(1):52. doi:10.1186/1476-069X-13-52

13. Che LF, Shao SF, Wang LX. Downregulation of CCR5 inhibits the proliferation and invasion of cervical cancer cells and is regulated by microRNA-107. Exp Ther Med. 2016;11(2):503-509. doi:10.3892/ etm.2015.291110.1126/science.aaa0355

14. Olar A, Aldape KD. Using the molecular classification of glioblastoma to inform personalized treatment. $J$ Pathol. 2014;232 (2):165-177. doi:10.1002/path.4282

15. Tastan Bishop Ö, Adebiyi EF, Alzohairy AM, et al. Bioinformatics education-perspectives and challenges out of Africa. Brief Bioinform. 2015;16(2):355-364. doi:10.1093/bib/bbu022

16. Melé M, Ferreira PG, Reverter F, et al. Human genomics. The human transcriptome across tissues and individuals. Science. 2015;348 (6235):660-665. doi:10.1126/science.aaa0355

17. Nannini M, Pantaleo MA, Maleddu A, Astolfifi A, Formica S, Biasco G. Gene expression profiling in colorectal cancer using microarray technologies: results and perspectives. Cancer Treat Rev. 2009;35(3):201-209. doi:10.1016/j.ctrv.2008.10.006

18. Wang M, Wang J, Liu J, et al. Systematic prediction of key genes for ovarian cancer by co-expression network analysis. J Cell Mol Med. 2020:10.1111/jcmm.15271. doi:10.1111/jcmm.15271.

19. Pyeon D, Newton MA, Lambert PF, et al. Fundamental differences in cell cycle deregulation in human papillomavirus-positive and human papillomavirus-negative head/neck and cervical cancers. Cancer Res. 2007;67(10):4605-4619. doi:10.1158/0008-5472.CAN-06-3619

20. Pappa KI, Polyzos A, Jacob-Hirsch J, et al. Profiling of discrete gynecological cancers reveals novel transcriptional modules and common features shared by other cancer types and embryonic stem cells. PLoS One. 2015;10(11):e0142229. doi:10.1371/journal.pone.0142229

21. den Boon JA, Pyeon D, Wang SS, et al. Molecular transitions from papillomavirus infection to cervical precancer and cancer: role of stromal estrogen receptor signaling. Proc Natl Acad Sci U S A. 2015;112(25):E3255-E3264. doi:10.1073/pnas.1509322112

22. Tang Z, Chenwei L, Kang B. GEPIA: a web server for cancer and normal gene expression profiling and interactive analyses. Nucleic Acids Res. 2017;10.1093/nar/gkx247.

23. Leek JT, Evan Johnson W, Parker HS, et al. sva: surrogate variable analysis. R package version 3.34.0. 2019.

24. Ritchie ME, Phipson B, Wu D, et al. limma powers differential expression analyses for RNA-sequencing and microarray studies. Nucleic Acids Res. 2015;43(7):e47. doi:10.1093/nar/gkv007

25. Szklarczyk D, Gable AL, Lyon D, et al. STRING v11: protein-protein association networks with increased coverage, supporting functional discovery in genome-wide experimental datasets. Nucleic Acids Res. 2019;47(D1):D607-D613. doi:10.1093/nar/gky1131

26. Zhang M, Zheng Y, Sun Y, et al. Knockdown of NEAT1 induces tolerogenic phenotype in dendritic cells by inhibiting activation of NLRP3 inflammasome. Theranostics. 2019;9(12):3425-3442. doi: $10.7150 /$ thno. 33178

27. Carlson M. org.Hs.eg.db: genome wide annotation for human. R package version 3.10.0. 2019.

28. Guangchuang Y, Wang L-G, Han Y, Qing-Yu H. clusterProfiler: an $\mathrm{R}$ package for comparing biological themes among gene clusters. OMICS. 2012;16(5):284-287. doi:10.1089/omi.2011.0118
29. Shannon P, Markiel A, Ozier O, et al. Cytoscape: a software environment for integrated models of biomolecular interaction networks. Genome Res. 2003;13(11):2498-2504. doi:10.1101/gr.1239303

30. UniProt Consortium. UniProt: a worldwide hub of protein knowledge. Nucleic Acids Res. 2019;47(D1):D506-D515. doi:10. 1093/nar/gky1049.

31. Sarfraz S, Hamid S, Ali S, Jafri W, Siddiqui AA. Modulations of cell cycle checkpoints during $\mathrm{HCV}$ associated disease. BMC Infect Dis. 2009;9(125). doi:10.1186/1471-2334-9-125

32. Li J, Sun L, Xu F, et al. Screening and identification of APOC1 as a novel potential biomarker for differentiate of Mycoplasma pneumoniae in children. Front Microbiol. 2016;7:1961. doi:10.3389/ fmicb.2016.01961

33. Liu CT, Min L, Wang YJ, Li P, Wu YD, Zhang ST. shRNA-mediated knockdown of KNTC1 suppresses cell viability and induces apoptosis in esophageal squamous cell carcinoma. Int J Oncol. 2019;54 (3):1053-1060. doi:10.3892/ijo.2019.4672

34. Diniz MG, Silva J, de Souza FT, Pereira NB, Gomes CC, Gomez RS. Association between cell cycle gene transcription and tumor size in oral squamous cell carcinoma. Tumour Biol. 2015;36(12):9717-9722. doi:10.1007/s13277-015-3735-1

35. Wolf M, Korja M, Karhu R, et al. Array-based gene expression, CGH and tissue data defines a 12q24 gain in neuroblastic tumors with prognostic implication. BMC Cancer. 2010;10(1):181. doi:10.1186/ 1471-2407-10-181

36. Kim YR, Chung NG, Kang MR, Yoo NJ, Lee SH. Novel somatic frameshift mutations of genes related to cell cycle and DNA damage response in gastric and colorectal cancers with microsatellite instability. Tumori. 2010;96(6):1004-1009.

37. Shen S, Kong J, Qiu Y, Yang X, Wang W, Yan L. Identification of core genes and outcomes in hepatocellular carcinoma by bioinformatics analysis. $J$ Cell Biochem. 2019;120(6):10069-10081. doi:10. $1002 / \mathrm{jcb} .28290$

38. Yan X, Wan H, Hao X, et al. Importance of gene expression signatures in pancreatic cancer prognosis and the establishment of a prediction model. Cancer Manag Res. 2018;(11):273-283. doi:10.2147/CMAR.S185205

39. Liu K, Kang M, Zhou Z, Qin W, Wang R. Bioinformatics analysis identifies hub genes and pathways in nasopharyngeal carcinoma. Oncol Lett. 2019;18(4):3637-3645. doi:10.3892/ol.2019.10707

40. Chan GK, Jablonski SA, Starr DA, Goldberg ML, Yen TJ. Human Zw10 and ROD are mitotic checkpoint proteins that bind to kinetochores. Nat Cell Biol. 2000;2(12):944-947. doi:10.1038/ 35046598

41. Keen N, Taylor S. Aurora-kinase inhibitors as anticancer agents. Nat Rev Cancer. 2004;4(12):927-936. doi:10.1038/nrc1502

42. Scaërou F, Starr DA, Piano F, Papoulas O, Karess RE, Goldberg ML. The ZW10 and rough deal checkpoint proteins function together in a large, evolutionarily conserved complex targeted to the kinetochore. J Cell Sci. 2001;114(Pt 17):3103-3114.

43. McMurry JA, Köhler S, Washington NL, et al. Navigating the phenotype frontier: the monarch initiative. Genetics. 2016;203(4):14911495. doi:10.1534/genetics. 116.188870

44. Mungall CJ, McMurry JA, Köhler S, et al. The monarch initiative: an integrative data and analytic platform connecting phenotypes to genotypes across species. Nucleic Acids Res. 2017;45(D1):D712-D722. doi:10.1093/nar/gkw1128 


\section{Publish your work in this journal}

OncoTargets and Therapy is an international, peer-reviewed, open access journal focusing on the pathological basis of all cancers, potential targets for therapy and treatment protocols employed to improve the management of cancer patients. The journal also focuses on the impact of management programs and new therapeutic

Submit your manuscript here: https://www.dovepress.com/oncotargets-and-therapy-journa agents and protocols on patient perspectives such as quality of life, adherence and satisfaction. The manuscript management system is completely online and includes a very quick and fair peer-review system, which is all easy to use. Visit http://www.dovepress.com/ testimonials.php to read real quotes from published authors. 